\title{
Análisis temporal de biomasa y stocks de carbono en un ecosistema de dehesa mediante imágenes Landsat, y su relación con factores climáticos
}

José Cáceres, M. Pilar Martín, y Javier Salas

\section{Resumen:}

Según la guía de buenas prácticas del Panel Intergubernamental de Expertos en Cambio Climático (IPCC), las técnicas de teledetección son adecuadas para la estimación de los sumideros de carbono a partir de la estimación de la biomasa. En este trabajo se ha utilizado una serie temporal de imágenes Landsat para estimar la cantidad de biomasa del pasto en una zona de dehesa al NE de la provincia de Cáceres (España) en el período 1985-2010. A partir de las imágenes de biomasa obtenidas se ha analizado la evolución de los stocks de carbono en la cubierta herbácea. Se realizaron muestreos de campo de biomasa, así como de radiometría, en cuatro parcelas ubicadas dentro de la zona de estudio para la calibración del modelo. Se aplicó un modelo de regresión lineal entre los datos de campo e índices espectrales para la estimación de biomasa a partir de las imágenes Landsat. El índice EVI obtuvo los mejores ajustes ( $R^{2}=0.79$ ). Se aplicó un factor de carbono de $50 \%$ de la biomasa para la estimación de los stocks de carbono en la cubierta herbácea. Los valores promedios de biomasa mostraron sensibilidad a los cambios extremos de las series temporales de precipitación y temperatura.

Palabras clave: Landsat; dehesa; índices espectrales; biomasa; stocks de carbono; variables climáticas

\section{Abstract:}

According to the Intergovernmental Panel on Climate Change Good Practice Guidance (IPCC GPG), remote sensing techniques are suitable for the estimation of carbon sinks, through the estimation of above ground biomass. In this work we used a Landsat time series to estimate grass biomass in a wooded grassland ecosystem 
located Northeast Cáceres (Spain) from 1985 to 2010. From the results of the estimation of biomass, we analyzed the evolution of carbon stocks in grasslands. Field sampling of biomass were performed, as well as field spectroscopy, in four plots located within the study site for model calibration. Linear regression models between field data and spectral indices were applied for estimating biomass from Landsat data. EVI was the best index for biomass estimation $\left(R^{2}=0.79\right)$. A carbon fraction of $50 \%$ of the biomass was applied for the carbon stock estimation. The average values of biomass were found to be sensitive to extreme changes in time series of precipitation and temperature.

Keywords: Landsat; spectral indices; biophysical variables; carbon stocks; climatic variables

José Cáceres ( josedavid.caceres@gmail.com), Facultad de Ciencias Espaciales (FACES), Universidad Nacional Autónoma de Honduras (UNAH), Edificio K2, Ciudad Universitaria, Tegucigalpa M.D.C.; M. Pilar Martín, Instituto de Economía, Geografía y Demografía (IEGD), Centro de Ciencias Humanas y Sociales (CCHS), Consejo Superior de Investigaciones Científicas (CSIC); Unidad Asociada Geolab; Javier Salas, Departamento de Geografía y Geología, Universidad de Alcalá (UAH); Unidad Asociada Geolab 


\section{INTRODUCCIÓN}

Las masas forestales juegan un papel fundamental en el ciclo del carbono, fijándolo de la atmósfera y almacenándolo en su biomasa, y constituyen, por ende, uno de los más importantes sumideros de carbono. Ante los compromisos suscritos por los países, incluido España, para la mitigación del cambio climático en la Convención Marco de Las Naciones Unidas sobre el Cambio Climático (CMNUCC), la cuantificación del balance de carbono se presenta como uno de los principales desafíos al permitir considerar la fijación de carbono como un objetivo más de la gestión forestal. Esto conlleva la estimación del carbono almacenado en la biomasa, así como el seguimiento de la evolución de estos stocks a diferentes escalas temporales.

La estimación de los stocks de carbono en zonas heterogéneas, como las dehesas, sabanas o bosques abiertos, utilizando sensores remotos, representa desafíos únicos debido a la presencia de dos estratos de vegetación con un comportamiento muy diferenciado, el pasto y las encinas (Todd et al. 1998).

Este estudio se basó en el uso de índices espectrales, derivados de imágenes Landsat, para estimar biomasa en una zona de dehesa en el momento de máxima actividad vegetativa, que corresponde con la época de primavera (marzo-mayo). A partir de una serie temporal de mediciones de campo e imágenes Landsat se ha generado un modelo empírico para la estimación de biomasa infiriendo, a continuación, la capacidad de fijación de carbono. Se ha puesto en relación la evolución temporal de esta variable con los cambios observados en los datos climáticos de estaciones meteorológicas localizadas en nuestra zona de estudio.

\section{ANTECEDENTES}

En la última década, la teledetección ha brindado información esencial sobre la variación espacial y temporal de las cubiertas vegetales, contribuyendo a la estimación de importantes variables biofísicas y bioquímicas. Hasta el momento se han aplicado con éxito enfoques empíricos basados en índices de vegetación que han obtenido un nivel de precisión satisfactorio para estimar importantes parámetros biofísicos de la vegetación como el contenido en clorofila (Blackburn 1998; Broge y Mortensen 2002) y nitrógeno (Hansen y Schjoerring 2003; Martin et al. 
2008). Otras aproximaciones basadas en el uso de modelos físicos de transferencia radiativa han demostrado igualmente su utilidad para la estimación de variables de interés como el contenido de agua de la vegetación (Trombetti et al. 2008; Yebra y Chuvieco 2009).

En este contexto se inscriben las actividades del proyecto Biospec (http:// www.lineas.cchs.csic.es/biospec/), que explora el uso de la teledetección próxima y remota para estimar diversos parámetros biofísicos de la vegetación en una zona de dehesa localizada al Noreste de la provincia de Cáceres (España). Para ello se ha realizado una intensa campaña de campo que ha permitido recopilar un conjunto muy importante de datos sobre el estado de la vegetación y sobre su comportamiento espectral, los cuales están siendo utilizados para calibrar y validar modelos empíricos y físicos para la estimación de variables biofísicas a partir de datos de teledetección obtenidos a diversas escalas espaciales y temporales.

En las últimas décadas la teledetección ha sido utilizada ampliamente para la estimación de biomasa en pasto (Azong Cho et al. 2007; Edirisinghe et al. 2012; Kogan et al. 2004; Malmstrom et al. 2009; Todd et al. 1998). A escala continental, sensores de baja resolución espacial, tales como NOAAAVHRR (Kogan et al. 2004) y MODIS (Muukkonen y Heiskanen 2007), han sido muy utilizados debido a su adecuada cobertura espacial y frecuencia de adquisición. Sin embargo, para cuantificar biomasa a escalas locales, es necesario contar con información obtenida por sensores con una resolución espacial más fina, como la obtenida por Landsat TM y ETM+ (Malmstrom et al. 2009; Meng et al. 2007; Todd et al. 1998). En este caso, el enfoque más frecuente consiste en relacionar índices de vegetación con datos de biomasa tomados en campo a través de modelos estadísticos (Azong Cho et al. 2007; Edirisinghe et al. 2012; Malmstrom et al. 2009; Meng et al. 2007; Muukkonen y Heiskanen 2007; Todd et al. 1998).

Para la estimación de los stocks de carbono a partir de la biomasa, la Organización de Naciones Unidas para la Agricultura y la Alimentación (FAO) propone aplicar una fracción de carbono (Marklund y Schoene 2006) cuyo valor varía entre el $45 \%$ y $53 \%$, utilizándose por defecto, generalmente, un valor de $50 \%$. Cuando se tiene información de la especie sobre la que se quiere estimar el contenido de carbono se pueden aplicar fracciones más acordes a la mismas (García et al. 2010). 


\section{3. ÁREA DE ESTUDIO}

El área de estudio se encuentra localizada en una zona de dehesa situada al NE de la provincia de Cáceres (Centro-Oeste de la Península Ibérica). El área propuesta cubre una extensión de 40.280,18 ha e incluye parte del territorio de 12 municipios (Figura 1). La definición del área se realizó utilizando como base la cartografía generada por el Sistema de Información sobre Ocupación del Suelo en España (SIOSE), identificando y seleccionando la zona correspondiente a la cobertura de dehesa.

Figura 1. Mapa de Localización del Área de Estudio

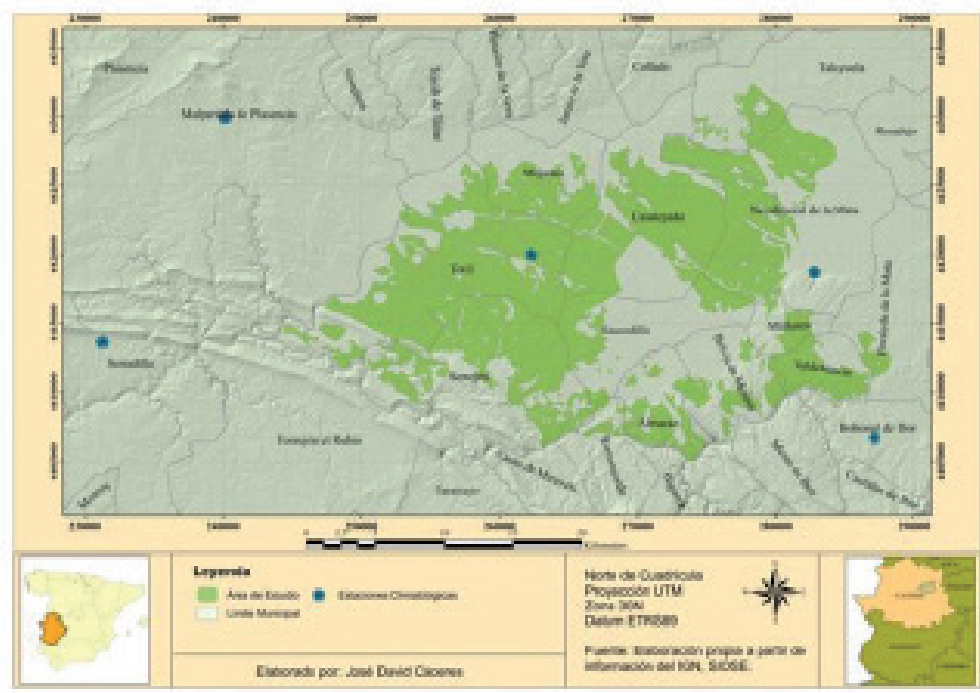

El relieve de la zona es poco accidentado, con una altitud media de $258 \mathrm{~m}$. La zona presenta una precipitación promedio anual de $645 \mathrm{~mm}$ y una temperatura media anual de $16.8^{\circ} \mathrm{C}$. Los meses más lluviosos son febrero y marzo, y los más secos, julio y agosto. Las temperaturas máximas se registran durante julio y agosto, y las mínimas en enero y febrero. Predominan los Lixisoles (sustratos no calizos del Mioceno) y la profundidad del suelo es mayor a $80 \mathrm{~cm}$. El tipo de vegetación natural corresponde a las series meso-mediterráneas de Pyro bourgeaneae - Querceto rotundifoliae quercetum, que ha sido transformada en praderas arboladas (encinas y especies herbáceas), incluidas algunas especies arbustivas (Cistus landanifer, Lavandula pedunculata y Ulex europaeus). El manejo de la tierra se centra en el pastoreo intensivo de vacuno. La densidad de árboles es de aproximadamente 20 árboles por hectárea, con una altura promedio de $8 \mathrm{~m}$ y un diámetro a la altura del 
pecho (DAP) promedio de $40 \mathrm{~cm}$.

\section{METODOLOGÍA}

Para la consecución de los objetivos planteados, se definió un esquema metodológico en 5 fases (Figura 2). En primer lugar, aunque en paralelo con las dos siguientes fases, se procedió a la selección y pre-procesamiento de las imágenes Landsat, así como al cálculo de diversos índices espectrales. La segunda y tercera fases incluyen el trabajo de campo en el que se realizaron mediciones de la variable biofísica de interés (biomasa), así como de la radiometría de la cubierta de pastizal. En la cuarta fase se generaron modelos empíricos para la estimación de la biomasa a partir de ajustes de regresión lineal entre índices espectrales obtenidos de la radiometría de campo y los valores de biomasa medidos en terreno en las mismas fechas. Utilizando estos modelos empíricos, se estimó la cantidad de biomasa a partir de los índices espectrales obtenidos con las imágenes Landsat. Esta cuarta fase se concluye con la estimación de los stocks de carbono a partir de los valores de biomasa. En la última fase se compara la evolución temporal de las variables estimadas con la de dos variables meteorológicas, la precipitación y la temperatura.

Figura 2. Descripción general del proceso de estimación de biomasa y carbono.

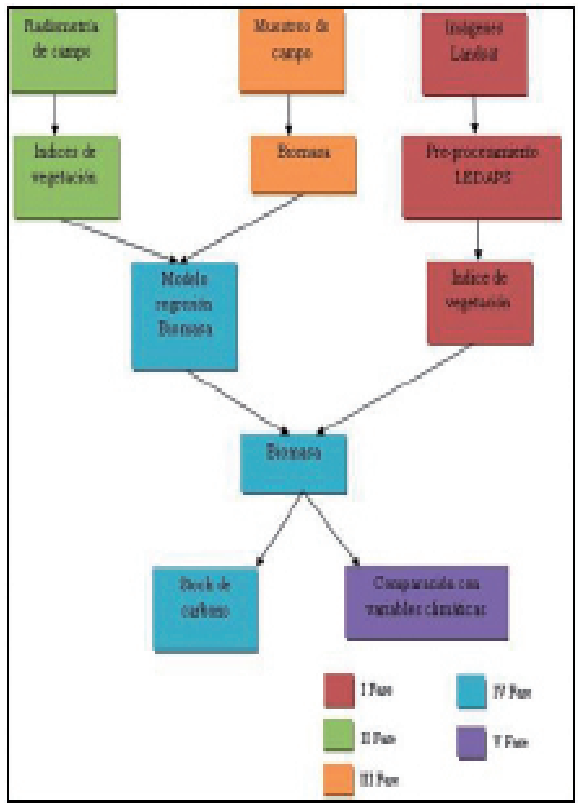




\subsection{Selección y pre-procesamiento de Imágenes Landsat}

Para este estudio se utilizó una serie histórica de la escena 202-032 adquirida por los sensores Enhanced Thematic Mapper Plus (ETM+) y Thematic Mapper (TM) a bordo del satélite Landsat. Las escenas fueron descargadas del servidor gratuito de imágenes del Servicio Geológico de los Estados Unidos (http://glovis. usgs.gov/).

Para el presente trabajo se han utilizado sólo las escenas libres de nubes de nuestra zona de interés disponibles en el catálogo de Glovis para los meses de primavera (marzo a mayo) desde la década de los 80 hasta la actualidad. Con el propósito de obtener la serie más completa posible se seleccionaron escenas tanto de Landsat 7 ETM+ como de Landsat 5 TM. Teniendo en cuenta las limitaciones mencionadas, finalmente se seleccionaron un total de 13 escenas desde 1985 a 2010 (Tabla 1).

Tabla1.Listado de escenas Landsat

\begin{tabular}{ll}
\hline Sensor & Fecha de Adquisición \\
\hline TM & 25 marzo 1985 \\
\hline TM & 12 abril 1987 \\
ETM+ & 25 mayo 2000 \\
ETM+ & 10 abril 2001 \\
ETM+ & 29 abril 2002 \\
\hline ETM+ & 18 mayo 2003 \\
\hline ETM+ & 1 marzo 2004 \\
\hline ETM+ & 7 mayo 2005 \\
\hline ETM+ & 24 abril 2006 \\
\hline ETM+ & 10 marzo 2007 \\
\hline ETM+ & 9 febrero 2008 \\
\hline ETM+ & 15 marzo 2009 \\
\hline TM & 11 abril 2010 \\
\hline
\end{tabular}

Una vez obtenidas las escenas a utilizar se procedió a realizar el pre-procesamiento de las imágenes, esto incluye la transformación de los niveles digitales (ND) originalmente captados por el sensor a valores de radiancia y reflectividad, incluyendo las oportunas correcciones del efecto atmosférico. Para este fin se utilizó la herramienta desarrollada por el Landsat Ecosystem Disturbance Adaptative Processing System (LEDAPS) (http://ledaps.nascom.nasa.gov/). 
LEDAPS es un proyecto de la NASA cuyo objetivo es cartografiar las perturbaciones, la regeneración y la conversión forestal permanente en Norteamérica (Masek et al. 2006). Entre las herramientas desarrolladas en el proyecto para el tratamiento y análisis de imágenes de satélite se encuentra el software LEDAPS preprocessing (http://ledaps.nascom.nasa.gov/tools/tools.html), originalmente desarrollado en la NASA Goddard Space Flight Center (GSFC) por Eric Vermote, Saleous Nazmi, Kutler Jonathan y Robert Wolfe. Esta herramienta se ha utilizado para el pre-procesamiento de todas las imágenes Landsat utilizadas en este trabajo.

El proceso llevado a cabo por la herramienta LEDAPS consiste en una línea de comandos, ejecutados secuencialmente, que constan de 4 pasos:

- Extracción de los metadatos del producto Landsat estándar y generación de los archivos de configuración para el resto del proceso. También genera la cabecera de los archivos para el programa de tratamiento digital ENVI (http://www. exelisvis.com/ProductsServices/ENVI/ENVI.aspx).

- Calibración radiométrica de Landsat-5 TM y Landsat-7 ETM+.

- Generación de una máscara de nubes utilizando el algoritmo Automated Cloud Cover Assesment (ACCA) (Irish et al. 2006).

- Corrección atmosférica.

En este trabajo la calidad de la corrección efectuada por la herramienta LEDAPS fue contrastada con datos de radiometría de campo obtenidos simultáneamente a la adquisición de una de las imágenes analizadas correspondiente al 30 de junio de 2010.

\subsection{Mediciones de Campo de Variables Biofísicas.}

Con el propósito de calibrar un modelo empírico para la estimación del contenido en biomasa del pasto a partir de las imágenes Landsat, se utilizaron los datos de terreno recopilados en el ámbito del proyecto BIOSPEC. Estos datos se refieren a un total de 12 parcelas de 25x25 m localizadas en la dehesa objeto de estudio. Estas parcelas fueron muestreadas cada 16 días haciendo coincidir el muestreo con el paso del satélite Landsat 5 desde marzo de 2009 hasta abril de 2011. De las 
12 parcelas disponibles se seleccionaron para este estudio las 4 que presentaban mayor densidad de vegetación, y de las fechas disponibles se utilizaron sólo las correspondientes a los meses de marzo y abril, que corresponden con el pico de verdor de la cubierta herbácea en esta zona. El objetivo de esta selección fue el de obtener datos de biomasa en parcelas/períodos con escasa o nula presencia de material senescente y suelo descubierto que pudieran interferir en la relación entre el dato de biomasa medido en terreno y la respuesta espectral de la vegetación captada por el sensor (Malmstrom et al. 2009).

Las medidas de biomasa se realizaron mediante muestreo destructivo. Se muestrearon 3 cuadrantes de $25 \times 25 \mathrm{~cm}$ distribuidos aleatoriamente en cada parcela. En cada cuadrante se recogió toda la vegetación cortándola a ras de suelo y se introdujo en una bolsa con cierre hermético, que fue pesada en el campo con una balanza de dos dígitos de precisión. Las muestras fueron transportadas al laboratorio donde se secaron en estufa a 60 grados centígrados durante 48 horas, después fueron pesadas de nuevo utilizando la misma báscula de campo. El contenido de biomasa del pasto se calculó como peso seco de la muestra dividido por el área del cuadrante.

\subsection{Mediciones de radiometría de campo}

En cada una de las parcelas en las que se tomaron muestras de biomasa de la vegetación, se realizaron también mediciones de radiometría sobre el terreno utilizando un espectro-radiómetro ASD FieldspecFR3 que recoge información entre 400 y 2500 nanómetros. Se realizaron dos transectos por parcela, uno NW-SE y otro NE-SW. Estas mediciones se realizaron en una franja horaria de \pm 2 horas respecto al mediodía solar, utilizando una óptica de $25^{\circ}$ y a una distancia aproximada de 1 metro sobre el dosel vegetal. Por cada transecto se tomaron una media de 10 mediciones, por lo que se contó finalmente con unos 20 espectros por parcela.

Los valores de reflectividad obtenidos con el ASD Fieldspec FR3, disponibles en intervalos de $1 \mathrm{~nm}$, fueron remuestreados a las bandas Landsat-TM utilizando el software ENVI que considera la función de respuesta espectral de cada banda. Con esta información se calcularon diversos índices espectrales propuestos en la literatura para estimar el contenido en biomasa de la vegetación. 


\subsection{Calibración de los modelos para la predicción de biomasa.}

Para la estimación de biomasa a partir de los datos de radiometría de campo y de las imágenes Landsat se emplearon en este trabajo los siguientes índices espectrales: Índice de Vegetación de Diferencia Normalizada (NDVI), cociente simple (SR), Índice de Vegetación Ajustado al Suelo (SAVI) e Índice de Vegetación Mejorado (EVI). Los índices se calcularon utilizando las siguientes expresiones (Marklund y Schoene 2006):

$$
\begin{aligned}
& N D V I=\frac{T M 4-T M 3}{T M 4+T M 3} \\
& S R=\frac{T M 4}{T M 3} \\
& S A V I=\left(\frac{T M 4-T M 3}{T M 4+T M 3+0.5}\right) \otimes(1+0.5) \\
& E V I=2.5 \text { 日 }\left(\frac{T M 4-T M 3}{T M 4+68 T M 3-7.5 \text { STM } 1+1}\right)
\end{aligned}
$$

Una vez calculados los índices a partir de los datos de radiometría de campo se realizó un análisis de correlación entre éstos y los datos de biomasa medidos en las 4 parcelas seleccionadas para las siguientes fechas: 23 de marzo, 7 y 24 de abril de 2009, 11 de abril de 2010, 30 de marzo y 14 de abril de 2011. El dato de campo para cada parcela fue un promedio de los 3 cuadrantes muestreados en cada una de ellas.

A partir del dato de la medición de biomasa en campo y del valor del índice de vegetación seleccionado se procedió a realizar un análisis de regresión lineal para obtener la ecuación de ajuste del modelo para la estimación de la biomasa. El modelo resultante se aplicó a las escenas Landsat seleccionadas para obtener los valores de biomasa para la serie temporal. Con este valor de biomasa se procedió a estimar el contenido de carbono aplicando una fracción de carbono del $50 \%$ del total de la biomasa.

\subsection{Información Climatológica}

Con el objetivo de analizar la posible relación existente entre la evolución anual de la biomasa con variables climatológicas, como precipitación y temperatura, se procedió a identificar las estaciones meteorológicas más cercanas al área de 
estudio. Se seleccionaron cinco estaciones próximas o incluidas en el área de estudio (Figura 1). Estos datos fueron solicitados a la Agencia Estatal de Meteorología (AEMET) para obtener las series históricas existentes.

Una vez obtenida la serie temporal de datos a utilizar, a partir de la fecha de adquisición de las escenas, se calculó la precipitación acumulada para dos meses (mes anterior y el propio mes de la toma de la imagen), así como también la temperatura máxima promedio para esos dos meses. Con estos datos, se realizó una comparación de la distribución temporal existente entre la precipitación y temperatura con la biomasa, de cara a identificar la posible influencia de las condiciones climáticas sobre las variables biofísicas analizadas.

\section{RESULTADOS Y DISCUSIÓN}

\subsection{Pre-procesamiento de imágenes Landsat}

Tal y como se ha descrito en el apartado de metodología, para comprobar la fiabilidad de la corrección radiométrica y atmosférica realizada por la herramienta LEDAPS se contó con datos de radiometría de campo para dos parcelas de calibración definidas por el proyecto BIOSPEC. Los valores de reflectividad medidos en el campo se compararon con los obtenidos utilizando la herramienta LEDAPS para la imagen Landsat 5 TM del 30 de junio de 2010. En la figura 3 se presentan las curvas espectrales obtenidas con los datos de campo y las extraídas de la imagen para las dos parcelas de calibración. Como se puede observar, las diferencias son mínimas entre ambos conjuntos de datos. De hecho, al realizar un análisis más cuantitativo de la información, se obtuvo que no existía una diferencia estadística significativa, existiendo una correlación fuerte entre los datos de campo y los de la imagen $\left(R^{2}=\right.$ 0.99), lo que indica que la herramienta LEDAPS resulta adecuada para el pre-procesamiento semi-automático de series temporales de imágenes Landsat. 
Figura 3. Comparación de espectros para las parcelas de calibración

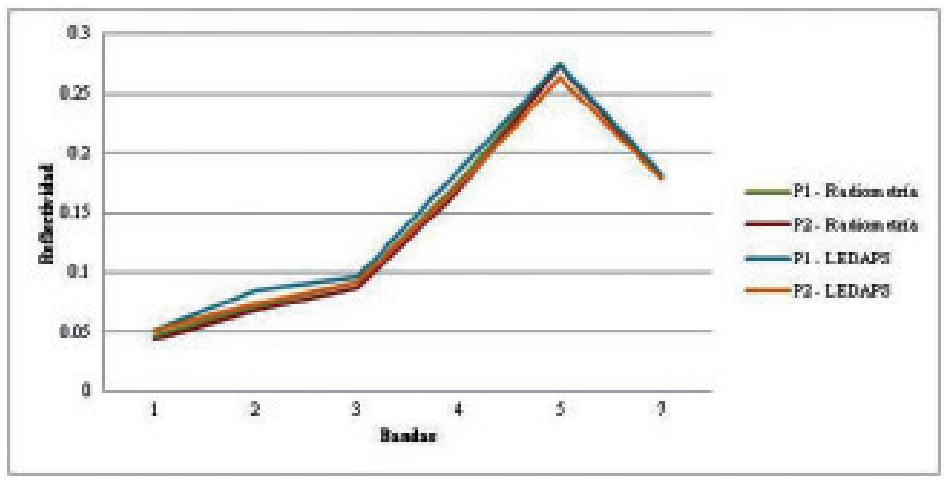

En general, se obtuvo una diferencia media entre los valores de reflectividad medidos en campo y los de satélite de 0.0061 para la parcela 1 y 0.0021 para la parcela 2. En la tabla 2 se presentan las diferencias porcentuales de variación observadas en cada banda del sensor para las dos parcelas de calibración. Los valores obtenidos mediante el uso de la herramienta LEDAPS fueron generalmente mayores que los obtenidos por radiometría de campo, si bien hubiera sido deseable realizar una validación más completa, que incluyera otras fechas/imágenes.

\subsection{Estimación de biomasa y carbono}

Los cuatro índices de vegetación utilizados para la estimación de la biomasa ofrecieron buenas correlaciones con la variable dependiente (biomasa medida en campo), con valores superiores a 0.73 (Tabla 3 ) y con un $p$-valor $<0.0001$. La mejor correlación se obtuvo con el $E V I$, con un $\mathrm{R}^{2}=0.79$, por lo cual fue éste el seleccionado para la estimación de la biomasa a partir de imágenes Landsat. 
Tabla 2. Diferencias relativas para cada banda entre reflectividad medida con radiometría de campo y

reflectividad obtenida de Landsat después de aplicar

\begin{tabular}{cccc}
\hline Bandass & $\begin{array}{c}\text { Longitud de } \\
\text { Onda Central }\end{array}$ & $\begin{array}{c}\text { \% de } \\
\text { Variación } \\
\text { Pareela 1 }\end{array}$ & $\begin{array}{c}\text { \% de } \\
\text { Variación } \\
\text { Parcela 2 }\end{array}$ \\
\hline $\mathbf{1}$ & 0.486572 & $\mathbf{1 0 . 1 4}$ & 16.71 \\
\hline $\mathbf{2}$ & 0.570932 & 17.98 & 9.53 \\
\hline $\mathbf{3}$ & 0.660975 & 5.95 & 4.97 \\
\hline $\mathbf{4}$ & 0.836878 & 5.63 & 2.30 \\
\hline $\mathbf{5}$ & 1.676651 & $\mathbf{0 . 1 2}$ & -3.70 \\
\hline $\mathbf{7}$ & 2.215476 & 1.79 & 0.53 \\
\hline
\end{tabular}

Tabla 3. Coeficientes de Correlación entre los valores de biomasa medidos en terreno y los índices espectrales calculados a partir de radiometría de campo

\begin{tabular}{ll}
\hline Indice & $\mathbf{R}^{2}$ \\
\hline NDVI & 0.7399 \\
SR & 0.7444 \\
SAVI & 0.7899 \\
EVI & 0.7939 \\
\hline
\end{tabular}

Los resultados obtenidos en nuestro estudio son similares a los obtenidos por Malmstrom et al. (2009) para la estimación de biomasa en una zona de pastizales en el valle de Sacramento, California. Los autores obtuvieron un $R^{2}=0.79$ y 0.83 para el SR y el NDVI respectivamente, realizando las mediciones durante marzo y abril. Todd et al. (1998) obtuvieron correlaciones algo inferiores utilizando modelos de regresión para zonas de pastoreo en un centro experimental en Colorado, estimando la biomasa mediante la aplicación de cuatro índices: GVI $\left(R^{2}=0.67\right), \mathrm{NDVI}$ $\left(R^{2}=0.66\right), R E D\left(R^{2}=0.64\right)$ y WI $\left(R^{2}=0.62\right)$. Kogan et al. (2004) también obtuvieron correlaciones inferiores $\left(\mathrm{R}^{2}=0.658\right)$ al utilizar el vegetation health $(\mathrm{VH})$, índice derivado del NDVI y la temperatura de brillo, para imágenes NOAA-AVHRR.

La ecuación de ajuste del modelo para la estimación de la biomasa a partir de los valores del EVI aparece en la figura 4. Esta función fue aplicada a las imágenes EVI calculadas a partir de la serie temporal Landsat, obteniéndose el valor de biomasa de la cubierta herbácea para cada uno de los años de estudio (Figura 5). 
Los valores de biomasa obtenidos a nivel de píxel para la serie temporal presentaron rangos desde valores muy cercanos a cero hasta $2,839.92 \mathrm{Kg} / \mathrm{ha}$, este valor máximo de la serie se registró en el año 2004. En general el valor medio de biomasa para cada píxel en toda la serie temporal fue de $772.30 \mathrm{Kg} / \mathrm{ha}$.

Malmstrom et al. (2009) obtuvieron valores un poco más elevados, registrando valores de hasta $3,640 \mathrm{Kg} / \mathrm{ha}$ para su zona de estudio y Kogan et al. (2004) obtuvieron un valor medio anual de 1,400 Kg/ha. En cambio Todd et al. (1998) obtuvieron valores inferiores, registrando un valor máximo de 1,600 Kg/ha.

Figura 4. Gráfico del Modelo Ajustado para la

Estimación de Biomasa

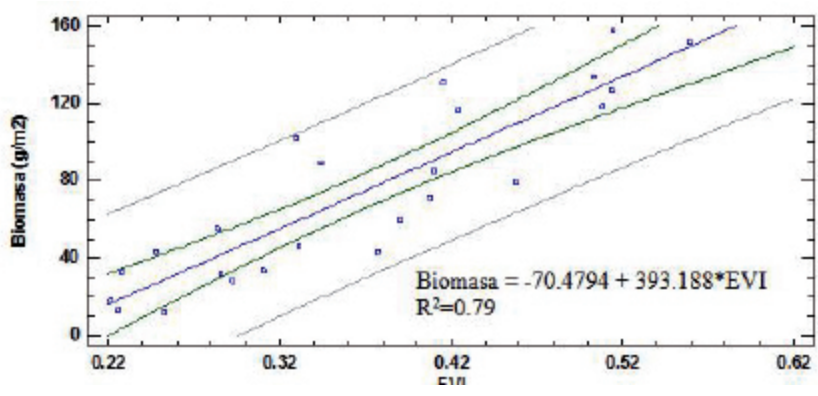

En general se observa una distribución espacial bastante uniforme de la biomasa en la zona de estudio, aunque aparece un sector al noreste en el que se registran valores más altos a lo largo de toda la serie. En el ámbito temporal se observa una mayor variabilidad con algunos años extremos como 2005, que presenta una disminución en la biomasa estimadas del 53\% respecto del promedio y el 2006 , con un aumento del $38 \%$ con respecto al promedio. 
Figura 5. Estimaciones de Biomasa del pasto obtenida a partir de imágenes Landsat para la zona de estudio, 1985-2010.
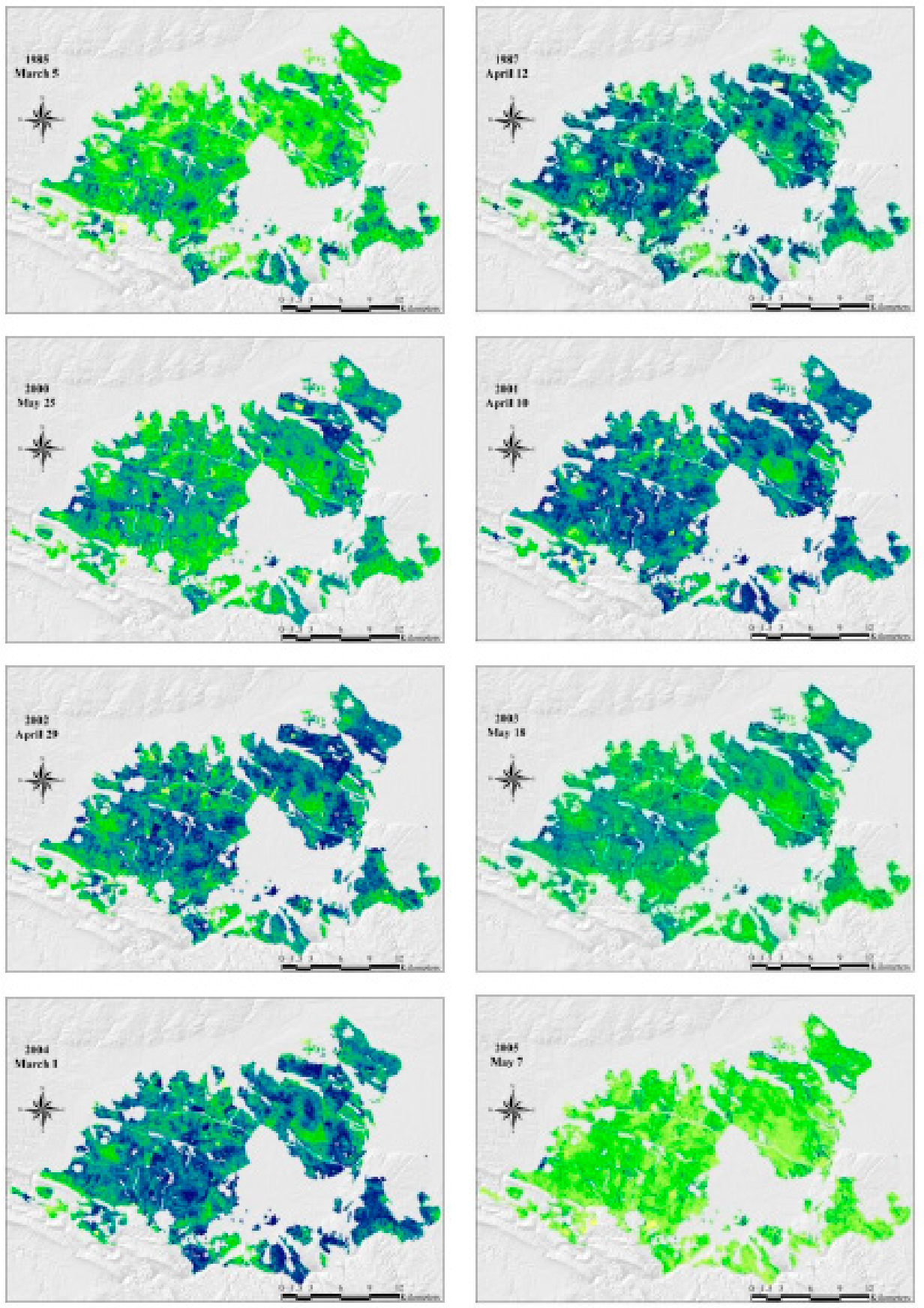

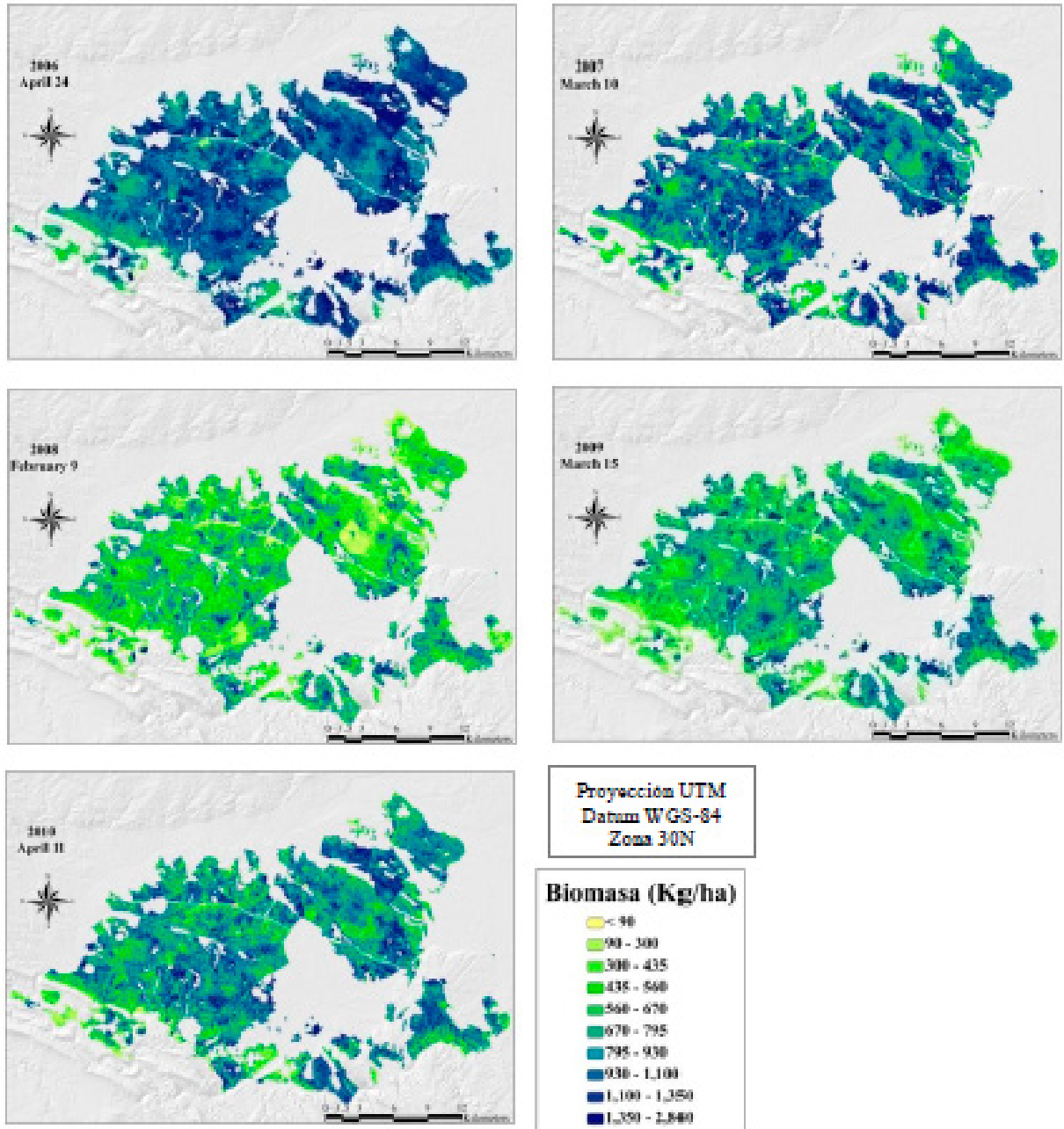

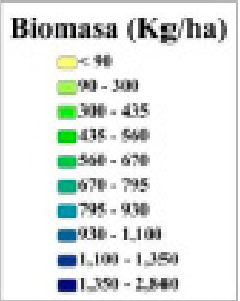

A partir de los valores de biomasa medios anuales calculados anteriormente, se procedió a aplicar la fracción de carbono del $50 \%$ del total de biomasa, para estimar el comportamiento de los stocks de carbono en el pasto en la zona de estudio. Los resultados se muestran en la tabla 4, teniendo en cuenta que la relación es directa entre la biomasa y el contenido de carbono, la tendencia en el comportamiento anual de las dos variables es exactamente la misma.

Para el período de estudio, el contenido de carbono en el pasto osciló entre $180 \mathrm{Kg} / \mathrm{ha}$ para el año 2005 y $536.09 \mathrm{Kg} / \mathrm{ha}$ para el 2006 . El valor medio de contenido de carbono para toda la serie temporal fue de $386.15 \mathrm{Kg} / \mathrm{ha}$. 
La mayoría de los estudios realizados en la estimación de los stocks de carbono se realizan sobre masas forestales ya que son sumideros con mayor capacidad y mayor estabilidad temporal que las zonas de pastos, por lo cual resulta difícil comparar los valoresobtenidos del contenido de carbono en nuestro ecosistema ya que serán inferiores a las cantidades presentes en masas boscosas.

Tabla 4. Cantidad promedio de biomasa y carbono estimado para la temporada de primavera en cada uno de los años analizados

\begin{tabular}{rrr}
\hline \multicolumn{1}{c}{ Año } & Biomasa Promedio (Kg/ha) & Contenido Carbone $(\mathrm{Kg} / \mathrm{has})$ \\
\hline 1985 & 545.54 & 272.77 \\
\hline 1987 & 850.45 & 425.23 \\
\hline 2600 & 704.12 & 352.06 \\
\hline 2001 & 935.32 & 467.66 \\
\hline 20012 & 885.86 & 442.93 \\
\hline 2003 & 679.51 & 339.76 \\
\hline 2604 & 925.01 & 462.50 \\
\hline 2005 & 360.01 & 180.00 \\
\hline 2006 & 1072.17 & 536.09 \\
\hline 2007 & 971.05 & 485.52 \\
\hline 2008 & 589.89 & 294.94 \\
\hline 2609 & 693.81 & 346.90 \\
\hline 2010 & 827.12 & 413.56 \\
\hline
\end{tabular}

\subsection{Relación con variables climáticas}

Los análisis realizados de comparación de muestras indican que no existen diferencias estadísticamente significativas entre los datos obtenidos para las cinco estaciones meteorológicas, registrando un $\mathrm{R}^{2}=0.95$ para las precipitaciones y un $R^{2}=0.99$ para las temperaturas.

Teniendo en cuenta la coincidencia existente para la serie histórica de datos entre las cinco estaciones analizadas, y para evitar el uso de estaciones que presentaban algunas lagunas en la serie temporal de los datos, se decidió tomar como referencia únicamente los datos de la estación de Navalmoral de la Mata, al presentar la serie más completa y estar ubicada dentro de nuestra zona de estudio (Figura 1).

Durante la serie temporal analizada (1985-2010) se observaron algunos años con comportamientos extremos. Así la precipitación fue muy inferior a la media en 2005 (43\%), coincidiendo con el año con menor valor promedio de biomasa 
( $360.01 \mathrm{Kg} / \mathrm{ha}$ ), y muy superior en el $2001,48 \%$ por encima de la media, año en el que se registró uno de los valores más altos de biomasa promedio $(935.32 \mathrm{Kg} / \mathrm{ha})$. El resto de los años muestran valores más cercanos a la media (Figura 6).

Figura 6. Serie temporal de precipitación y valor medio de biomasa estimada para primavera en la zona de estudio

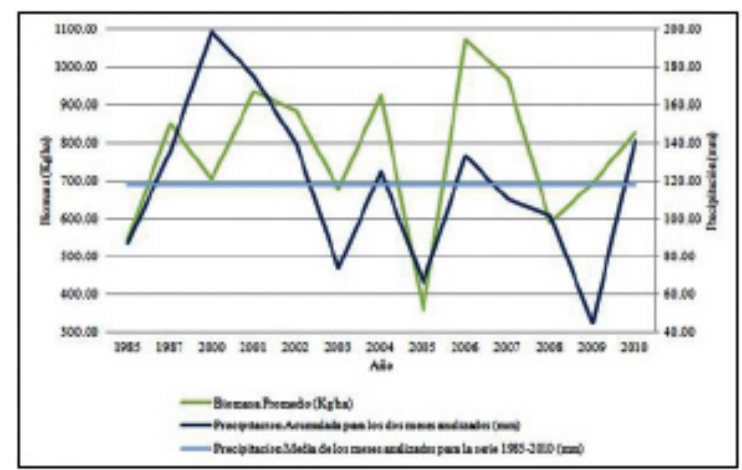

La biomasa mostró correlaciones bajas con las precipitaciones, aunque puede observarse una evolución temporal similar, con incrementos y descensos en los mismos años, a excepción de los años 2000 y 2009, en los que estas variables experimentan comportamientos opuestos. El 2009 fue un año especialmente seco, pero la imagen se adquirió poco después de registrarse precipitación en la zona de estudio, aunque en el conjunto de los dos meses utilizados en la comparación la cantidad de precipitación registrada fue muy baja. La divergencia en el año 2000 parece estar relacionada con la fecha de adquisición de la imagen ( 25 de mayo), en la que probablemente el pasto estaría notablemente seco.

Como en el caso de las precipitaciones, la temperatura también ofreció bajos valores de correlación con el contenido de biomasa. No obstante, salvo alguna excepción, se observa una clara tendencia inversa entre ambas variables, de manera que de acuerdo a lo observado, temperaturas más altas conllevan menores contenidos de biomasa (Figura 7). 
Figura 7. Serie temporal de temperatura máxima y valor medio de biomasa estimada para primavera en la zona de estudio

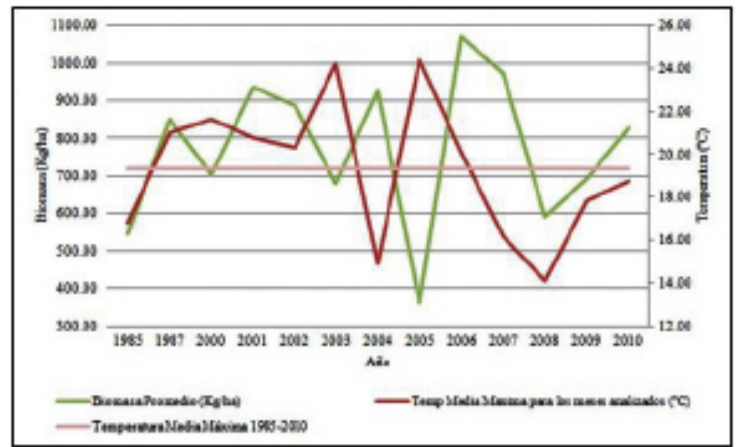

\section{CONCLUSIONES}

En este estudio hemos investigado el uso de índices espectrales derivados de los sensores TM y ETM+ a bordo del satélite Landsat para la estimación de biomasa y stocks de carbono del pasto en una zona de dehesa, así como la importancia de contar con información medida en campo para calibrar los modelos de estimación a partir de regresiones simples. Las imágenes Landsat fueron corregidas radiométricamente de forma automática utilizando la herramienta LEDAPS antes del cálculo de los índices espectrales y se obtuvo que, para la predicción de biomasa en pastos, la utilización de un índice como el EVI brinda un modelo más robusto $(\mathrm{R} 2=0.79)$ frente a otros índices más tradicionales como el NDVI, siendo este valor comparable con resultados obtenidos por otros autores que han empleado una metodología similar (Malmstrom et al. 2009; Todd et al. 1998). Estos datos ponen de manifiesto el interés de utilizar imágenes de satélite para la estimación de variables biofísicas, que de otra manera requerirían de costosos muestreos de campo, inabordables para territorios extensos.

Resalta el hecho de que la variable analizada es sensible a los cambios extremos en las condiciones climáticas, presentando sus valores más bajos en el año en que se registró la menor cantidad de precipitación y a su vez la mayor temperatura máxima (2005) en la zona, lo cual tuvo un impacto directo en la cantidad de biomasa. 
Aunque la serie temporal analizada registra importantes lagunas debidas a la inexistencia de imágenes Landsat en el catálogo Glovis, parece registrarse una ligera tendencia de disminución del contenido de biomasa en la serie temporal analizada, que se corresponde con una tendencia Figura 7. Serie temporal de temperatura máxima y valor medio de biomasa estimada para primavera en la zona de estudio similar en el registro de precipitaciones. La tendencia hacia una menor cantidad de lluvia limitaría en gran medida la biomasa del pasto y, por ende, reduciría su capacidad de fijar carbono, tal y como se observó en el año con menor cantidad de precipitación dentro de la serie temporal.

\section{Agradecimientos}

Agradezco a la Fundación Carolina por la beca otorgada para desarrollar el Máster en Tecnologías de la Información Geográfica en la Universidad de Alcalá, del cual se desprende este trabajo. Al Servicio Geológico de los Estados Unidos (USGS) por la disponibilidad de las imágenes Landsat. A Jeffrey Masek por facilitar el acceso a la herramienta de pre-procesamiento de LEDAPS y a la Agencia Estatal de Meteorología (AEMET) por la cesión de los datos meteorológicos. Este trabajo se ha realizado en el contexto de las actividades del proyecto BIOSPEC (CGL200802301/CLI) financiado por el Ministerio de Ciencia e Innovación español.

\section{BIBLIOGRAFÍA}

- $\quad$ Azong Cho, M., Skidmore, A., Corsi, F., van Wieren, S., \& Sobhan, I. (2007). Estimation of green/herb biomass from airborne hyperspectral imagery using spectral indices and partial least squares regression. International Journal of Applied Earth Observation and Geoinformation, 9, 414-424

- Blackburn, G.A. (1998). Quantifying chlorophylls and carotenoids at leaf and canopy scales; an evaluation of some hyperspectral approaches. Remote Sensing of Environment, 6, 273-285

- Broge , N.H., \& Mortensen, J.V. (2002). Deriving green crop area index and canopy chlorophyll density of winter wheat from spectral reflectance data. Remote Sensing of Environment, 81, 45-57 
- Edirisinghe, A., Clark, D., \& Waugh, D. (2012). Spatio-temporal modelling of biomass of intensively grazed perrennial dairy pastures using multispectral remote sensing. International Journal of Applied Earth Observation and Geoinformation, 16, 5-16

- García, M., Riaño, D., Chuvieco, E., \& Danson, F.M. (2010). Estimating biomass carbon stocks for a Mediterranean forest in central Spain using LiDAR height and intensity data. Remote Sensing of Environment, 114, 816-830

- Hansen, P.M., \& Schjoerring, J.K. (2003). Reflectance measurement of canopy biomass and nitrogen status in wheat crops using normalized difference vegetation indices and partial least squares regression. Remote Sensing of Environment, 86, 542-553

- Irish, R., Barker, J., Goward, S., \& Arvidson, T. (2006). Characterization of the Landsat-7 ETM+ Automated Cloud Cover Assessment (ACCA) Algorithm. Photogrammetric Engineering \& Remote Sensing, 72, 1179-1188

- Kogan, F., Stark, R., Gitelson, A., Jargalsaikhan, L., Dugrajav, C., \& Tsooj, S. (2004). Derivation of pasture biomass in Mongolia from AVHRR-based vegetation health indices. , 25, 2889-2896Marklund, L.G., \& Schoene, D. (2006). Global assessment of growing stock, biomass and carbon stock. In FAO (Ed.), Forest resources assessment programme 2005. Working paper 106. Rome (Italy): Food and Agriculture Organization of the United Nations (FAO)

- Martin, M.E., Plourde, L.C., Ollinger, S.V., Smith, M.L., \& McNeil, B. (2008). A generalizable method for remote sensing of canopy nitrogen across a wide range of forest ecosystems. Remote Sensing of Environment, 12, 3511-3519

- Masek, J.G., Vermote, E., Saleous, N., Wolfe, R., Hall, F., Huemmrich, K., Gao, F., Kutler, J., \& Lim, T.-K. (2006). A Landsat Surface Reflectance Dataset for North America, 1990-2000. Geoscience and Remote Sensing Letters, 3, 68-72

- Meng, Q., Cieszewski, C., Madden, M., \& Borders, B. (2007). A linear mixedeffects model of biomass and volume of trees using Landsat ETM+ images. Forest Ecology and Management, 244, 93-101 
- Muukkonen, P., \& Heiskanen, J. (2007). Biomass estimation over a large area based on standwise forest inventory data and ASTER and MODIS satellite data: A possibility to verify carbon inventories. Remote Sensing of Environment, 107, 617-624

- Todd, S.W., Hoffer, R.M., \& Milchunas, D.G. (1998). Biomass estimation on grazed and ungrazed rangelands using spectral indices. International Journal of Remote Sensing, 19, 427-438

- Trombetti, M., Riaño, D., Rubio, M.A., Cheng, Y.B., \& Ustin, S.L. (2008). Multitemporal vegetation canopy water content retrieval using Artificial Neural Networks for the USA. Remote Sensing of Environment, 112, 203-215

- Yebra, M., \& Chuvieco, E. (2009). Linking ecological information and radiative transfer models to estimate fuel moisture content in the Mediterranean region of Spain : Solving the ill-posed inverse problem. Remote Sensing of Environment, $113,2403-2411$ 